\title{
IoT Based Diabetes Detection through Iris Image Analysis
}

\author{
Shreeja A ${ }^{1}$, Ms. R Monika², Lolla BNV Sai Rama Pradeep ${ }^{3}$, N Vamsi Sandeep ${ }^{4}$, D HamsaVardhan ${ }^{5}$ \\ Department of Electronics \& Communication Engineering \\ SRM Institute of Science \& Technology, SRM Nagar, Kattankulathur, Tamil Nadu 603203. \\ ashreeja99@gmail.com ${ }^{1}$,monikar@srmist.edu.in²,pradeeplolla555@gmail.com ${ }^{3}$, \\ vamsisandeep0@gmail.com ${ }^{4}$, hamsavardhan99@gmail.com ${ }^{5}$
}

\begin{abstract}
Diabetes is generally caused due to high blood sugar levels. There are two types of this polygenic disease, type one and type two. Type two is the most typical style of polygenic disease. Polygenic disease causes severe health issues that embody cardiovascular disease, nephrosis, and eye issues. Iridodiagnosis is a branch of science that consists of varied algorithms and is developed for image quality assessment, Iris segmentation, Iris Normalization, Feature extraction, and classification. The developed system uses GLCM (Gray Level Co-occurrence Matrix) to obtain texture options and for feature classification, the SVM algorithm is employed. This approach is way quicker and efficient. Texture features were analyzed by using ThingSpeak.
\end{abstract}

Keywords - Iris, Segmentation, Normalization, GLCM texture features, SVM algorithm.

\section{INTRODUCTION}

Diabetes is a metabolic condition that occurs when the body is unable to produce the hormone insulin, or the insulin released can not be used efficiently by the body, resulting in a rise in the amount of blood glucose in the body. The hormone Insulin is produced in the pancreas by the beta cells and helps to transport glucose to the body cells from the bloodstream. Type I diabetes arises when the immune system attacks the pancreatic beta cells, resulting in small quantities of insulin released by the pancreas. The most common type of diabetes characterised by hyperglycemia due to low insulin production and ineffectiveness of the body in reacting to insulin is type II diabetes. The iris is an internal organ of the eye located behind the cornea. It regulates the pupil's size, and it controls the amount of light that enters the eye. The iris patterns are different for both eyes. The study of the iris is known as iridology.

The iris is sort of a map of the body. Changes in organs are mirrored in specific elements of the iris. It consists of sixty sectors and each of them deals with an indoor organ of the physique $[2,3]$. We'll be discussing the condition of the pancreas through the iris. An iridology chart offers the placement of the indoor organ on the iris. The main objective of irido diagnosis is to gather information about underlying diseases.

Arcus senilis is a whitish bow formed or ring-shaped deposit within the membrane. It is generally associated with coronary heart disease (CHD). It is additionally recognized as a symptom of hyperlipidemia. Iridologists believe that the grey or whitish deposit on the iris is a sign of the presence of steroid alcohol or cornea unwellness. This non-invasive and

Vol. 4 (8), February 2021, www.ijirase.com straightforward automation system is developed to find cholesterol presence [13].

ThingSpeak is an IoT analytics software application for aggregating, visualizing and evaluating live data sources in the cloud. ThingSpeak offers immediate visualization of data added to ThingSpeak through the computers. With the opportunity to perform MATLAB implementation in ThingSpeak, the data can be evaluated and interpreted online.

\section{PROPOSED APPROACH}

The process includes six steps which are 1) Iris image acquisition 2) Iris image segmentation 3) Iris image normalization 4) ROI Extraction 5) Feature extraction 6) Feature classification.

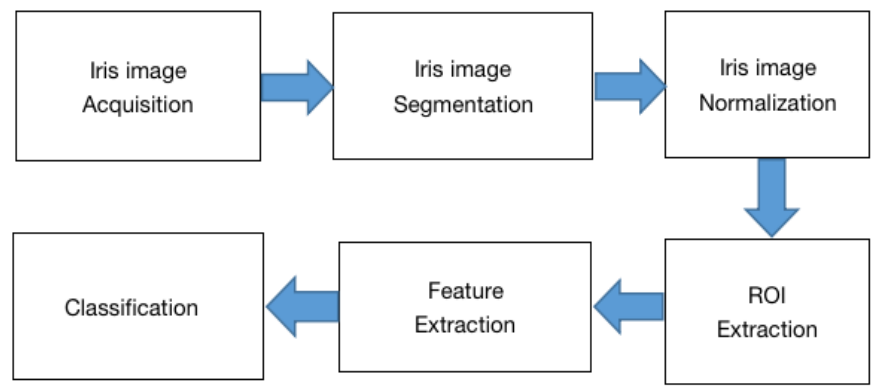

Fig 1. Methodology

1) Iris image acquisition:

An picture of the eye to be studied must first be collected in digital form appropriate for examination. With the assistance of some sensors, eye images are collected and are stored in 
the database. The recorded eye images for image processing must be of good quality and high resolution. Files from the CASIA database have been collected.

Pictures of eyes have to be captured clearly with specific cameras and stored in the database, containing both Normal and abnormal iris.

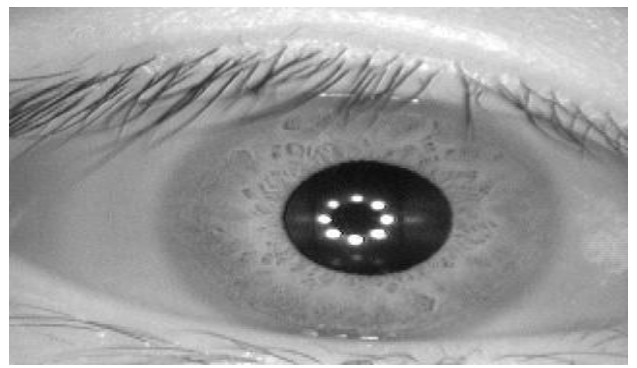

Fig 2. Iris image

2) Iris image segmentation:

Segmentation is performed to seek out the iris' inner and outer boundaries [4]. The proposed approach uses the canny edge detection rule [12] for the segmentation of Iris. The Canny edge detection rule involves four steps. The initial step is termed as a smoothening step. During this step, the operator softens the image to get rid of the noise. Gaussian Filters are accustomed to eliminate the unwanted noise occurring because of the eyelids and the eyelashes. Ironed pictures are filtered using Sobel- kernel operator in each horizontal and vertical direction to obtain the first derivative to calculate gradients. High Gradient magnitude means that the colors are dynamical, implying an edge. In the nonmaximum suppression step, the operator looks for native maxima in its neighborhood. It uses 2 thresholds (i.e. minimum and most value), which is often known as physical phenomenon thresholding. Edges with intensity gradient over the most worth are thought of to be true edges, those below minimum worth are discarded [15].

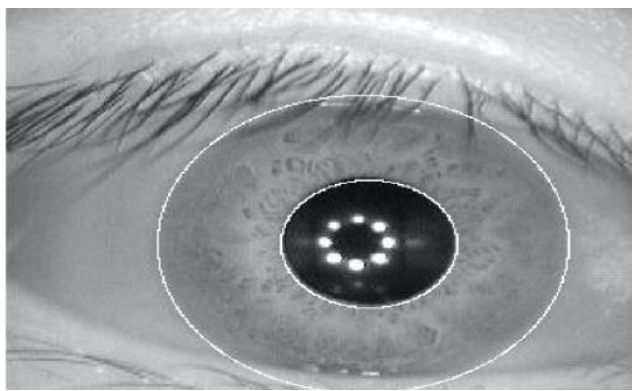

Fig 3. Segmented iris image

Vol. 4 (8), February 2021, www.ijirase.com
3) Iris image normalization:

The Iris orientation changes from person to person, so as to get a typical illustration for all normalization is critical. Normalization is needed to convert circular iris patterns into rectangular shapes. Daugman's rubber sheet model [14] is employed for the iris standardization method. It converts the circular iris region to a pair of polar coordinates $(r, \theta)$ [5] where $r$ lies in the interval $[0,1]$ and $\theta$ lies in the range of $[0,2 \Pi]$.

The pupil centre is taken into account as the centre, and radial vectors are passed through the iris region using Daugman's rubber sheet model to normalise the iris region. A variety of information points that are selected on any radial line reflect the radial resolution. Due to the variety of radial lines going around the iris field, the angular resolution is highlighted.

After obtaining the normalized iris region, the region is unwrapped by selecting a relentless range of points on every radial line and manufacturing a 2D array with vertical dimensions of radial resolution and horizontal dimensions of angular resolution. To forestall non-iris region information, another 2D array is formed for marking eyelashes, and eyelids detected within the segmentation stage are discarded.

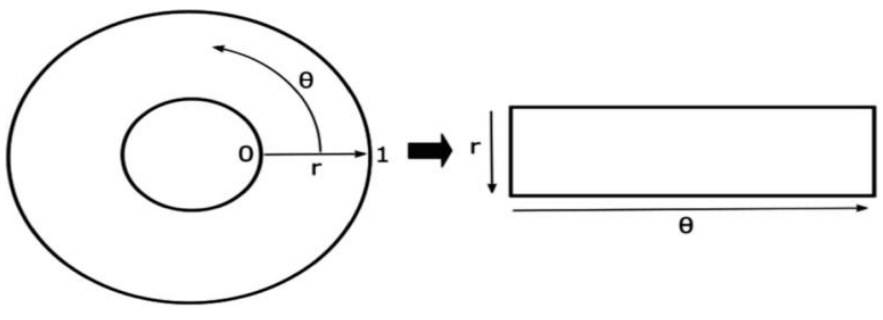

Fig 4. Daugman's rubber sheet model

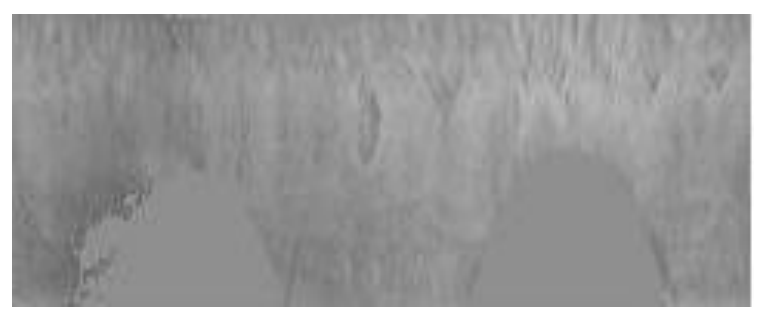

Fig 5. Normalized iris image

Normalized iris image is divided into four parts row-wise. In that, $30 \%$ normalization of the polar part is used for 
cholesterol presence detection [13], it is identified based on the iris chart. Threshold values and also the statement concerning the condition of the normal and arcus iris are shown below. From the bar graph and OTSU's methodology, the decidability or threshold worth to differentiate between traditional eyes and eyes with cornea Lipids is 139. If the cluster average is a smaller amount than this threshold, this suggests that the attention is the traditional eye and if it's above 139, then the attention may be detected as an eye with cornea Lipids.

To improve the iris image contrast, a stage of bar graph effort is required. Grey levels vary from 0 to 255 . We have to calculate the PDF and also the CDF of the image. PDF helps in calculating the probability of each pixel value of an image and CDF gives the cumulative sum of these values. An iris, whose pixels occupy a whole variety of grey levels, can have high contrast.
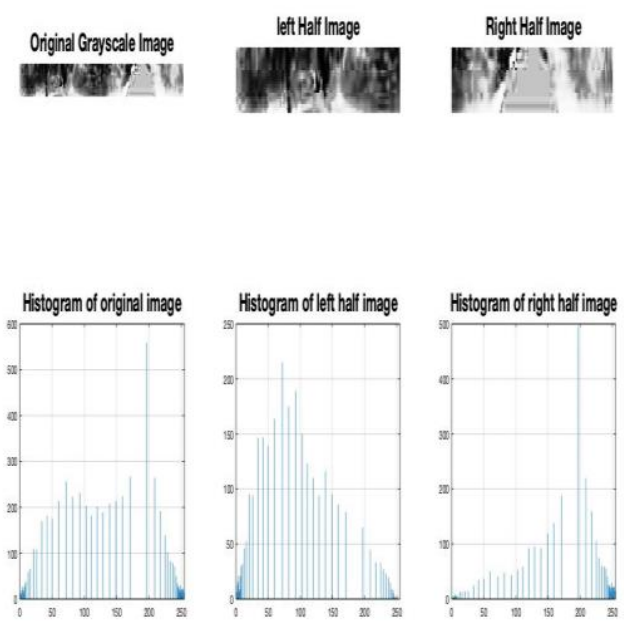

Fig 6. Histogram Analysis of 30\% Normalized Image

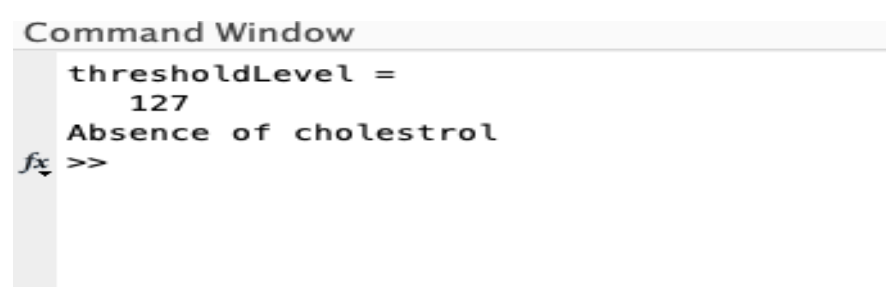

Fig 7. Threshold Level

4) ROI Extraction:

ROI Extraction, however, involves cropping a specific portion of the normalized iris image [1]. Each ROI may be a square region of mounted size. The scale of the ROI varies

Vol. 4 (8), February 2021, www.ijirase.com from seventeen to forty-nine pixels. Here we extract the pancreas section from the normalized image. The liver section ranges from $235^{\circ}$ to $242^{\circ}$ with a scale of iris radius of 0.36 to 1.0 in the right eye iris.

The pancreas is located in the iris's right eye in the third quarter of the eyeball at the region 06.45 to 07.15 with a depth of $0.4-0.6$ from the center [18].
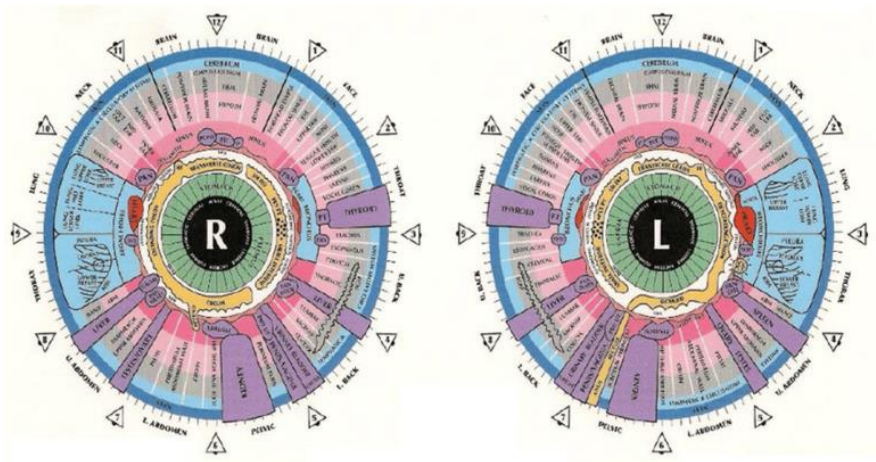

Fig 8. Iridology map

\section{5) Feature extraction:}

The feature is a performance of one or a lot of measurements. The GLCM $[\mathbf{1 0}, \mathbf{1 6}]$ is a methodology for extracting texture information from the image. It defines the connection between two neighbouring pixels in a grayscale image. The primary picture element is named as the reference picture element and also the second is named the neighbouring picture element. It describes the frequency of one grey level showing in a very mere relationship with another grey level. The matrix is adjusted to support the gap between pixels and also the angle. Features extracted are unit energy, contrast, correlation, homogeneity, and energy.

Contrast: Measures the spatial frequency of the image.

$$
\sum_{i, j}|i-j|^{2} p(i, j)
$$

Correlation: Measures the linear dependence of grey levels of neighboring pixels.

$$
\sum_{i, j} \frac{(i-\mu i)(j-\mu j) p(i, j)}{\sigma_{i} \sigma_{j}}
$$

Energy: Measures the feel uniformity.

$$
\sum_{i, j} p(i, j)^{2}
$$

Homogeneity: Measures the variations in image intensity.

$$
\sum_{i, j} \frac{p(i, j)}{1+|i-j|}
$$


6) Feature classification :

\section{SUPPORT VECTOR MACHINE (SVM)}

Support vector machines (SVMs) are strong but simple supervised algorithms for machine learning which are used for classification and regression. However, they are usually used in classification issues. SVM's were first developed in the 1960s but then improved in the 1990s. Similar to other machine learning methods, SVM's have their own special method of execution.

SVM is of two types:

Linear SVM: Linear SVM is used for linearly separable data, implying that if a dataset can be divided into two classes with a single straight line, it is considered linearly separable data, and classifier is considered a linear SVM classifier.

Non-linear SVM: Non-linear SVM is used for non-linear data, which implies that if a straight line can not categorize a sample, the data is considered non-linear data and the classifier used is considered non-linear SVM [4].

The following are essential terms in SVM

- Support Vectors - Data Points are considered to help vectors and are nearest to the hyperplane. With the aid of these data points, a dividing line is established.

- Hyperplane - It is a plane of judgment or space that a group of objects splits with various groups.

- Margin - The distance between two lines on the data points of different groups can be described. It can be measured as the perpendicular angle between the line and the support vectors. Big margins are called healthy margins, while low margins are known as poor margins.

SVM's key objective is to partition the datasets into classes to find a maximal marginal hyperplane (MMH) that can be achieved in the following two phases- First, iteratively, SVM must create a hyperplane that segregates classes in the best possible way. Then it will choose the hyperplane which properly separates the classes.

Vol. 4 (8), February 2021, www.ijirase.com

\section{ThingSpeak IoT:}

ThingSpeak helps to store, display and evaluate feeds of live data in the cloud. Suitable TimeStamps Should be Created before Uploading it into the cloud.

ThingSpeak's main features include:

- Quickly configure devices that can submit data to ThingSpeak using common IoT protocols.

- Visualize the data on the camera in real-time.
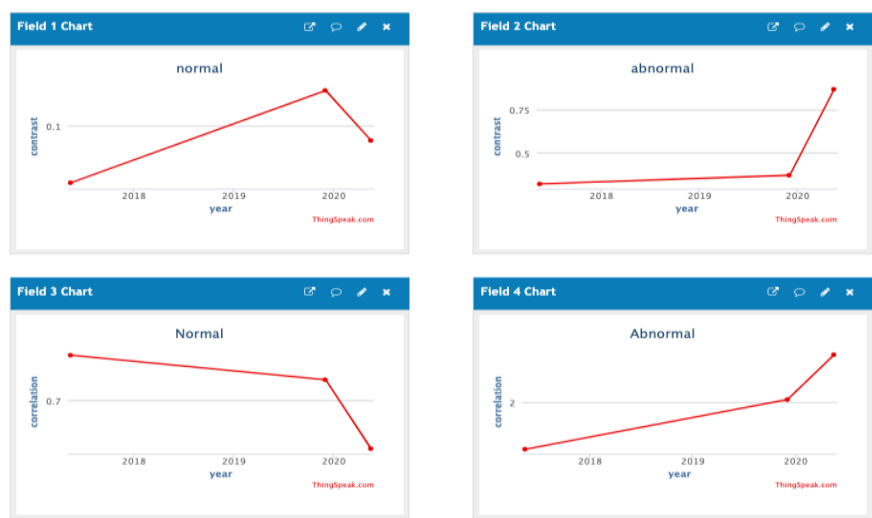

Fig 8. Texture Feature Analysis Using ThingSpeak IoT
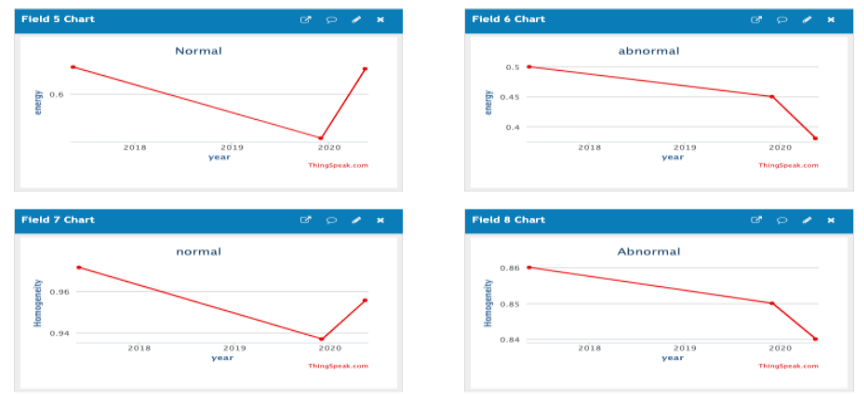

Fig 9. Texture Feature Analysis Using ThingSpeak IoT

\section{CONCLUSION}

This approach is reliable and efficient in carrying out the function of recommending an iris diagnosis and authentication. This would improve the diagnostic phase and give it more trust. The iris is very useful for human authentication and recognition because of its unique and consistent spatial patterns. Such successful iris detection is used to recognize people and verify whether iris is impaired and distinguish the damaged portion of the human eye. Since every part of the iris reflects the various areas of the human body, it will be used to diagnose the different diseases without any more damage to the human body. 


\section{REFERENCES}

1. Doss, S., Paranthaman, J., Gopalakrishnan, S., Duraisamy, A., Pal, S., Duraisamy, B., ... \& Le, D. N. (2021). Memetic Optimization with Cryptographic Encryption for Secure Medical Data Transmission in IoT-Based Distributed Systems. CMC-COMPUTERS MATERIALS \& CONTINUA, 66(2), 1577-1594.

2. Prasad, J., Patel, D., Jadhav, M., \& Deshmukh, R. (2016). IRIS BASED MEDICAL ANALYSIS BY GEOMETRIC DEFORMATION.

3. Chaskar, U. M., \& Sutaone, M. S. (2012, January). On a methodology for detecting diabetic presence from iris image analysis. In 2012 International Conference on Power, Signals, Controls and Computation (pp. 1-6). IEEE.

4. Moradi, P., Nazer, N., Ahmadi, A. K., Mohammadzade, H., \& Jafari, H. K. (2018, November). Discovering Informative Regions in Iris Images to Predict Diabetes. In 2018 25th national and 3rd International Iranian Conference on Biomedical Engineering (ICBME) (pp. 1-6). IEEE.

5. Tathe, M. P. B., \& Patil, M. D. M. (2015). Analysis of health condition based on iris image. International Journal of Innovation in Engineering, Research and Technology [IJIERT], 2015(1), 1-4.

6. Padmasini, N., Umamaheswari, R., Kalpana, R., \& Sikkandar, M. Y. (2020). Comparative study of iris and retinal images for early detection of diabetic mellitus. Journal of Medical Imaging and Health Informatics, 10(2), 316-325.

7. Mir, N., Khan, M. A., \& Hussain, M. G. (2020). Portable smart healthcare solution to eye examination for diabetic retinopathy detection at an earlier stage. In Innovation in Health Informatics (pp. 305-322). Academic Press.

8. Aminah, R., \& Saputro, A. H. (2019, September). Diabetes prediction system based on iridology using machine learning. In 2019 6th International Conference on Information Technology, Computer and Electrical Engineering (ICITACEE) (pp. 1-6). IEEE.

9. Adelina, D. C., Sigit, R., Harsono, T., \& Rochmad, M. (2017, September). Identification of diabetes in pancreatic organs using iridology. In 2017 International Electronics Symposium on Knowledge Creation and Intelligent Computing (IES-KCIC) (pp. 114-119). IEEE.
10. Reddy, R. P. K., Nagaraju, C., \& Reddy, I. R. (2016). Canny scale edge detection.

11. Canny, J. (1986). A computational approach to edge detection. IEEE Transactions on pattern analysis and machine intelligence, (6), 679-698.

12. Ramlee, R. A., \& Ranjit, S. (2009, April). Using iris recognition algorithm, detecting cholesterol presence. In 2009 International Conference on Information Management and Engineering (pp. 714-717). IEEE.

13. Bendre, M. R., \& Shivarkar, S. A. (2012). An Improved Approach for IRIS Authentication System by Using Daugman's Rubber Sheet Model, Segmentation, Normalization and RSA Security Algorithm. Journal of Computer Technology and Electronics Engineering, 1(3), 102-107.

14. Mohanaiah, P., Sathyanarayana, P., \& GuruKumar, L. (2013). Image texture feature extraction using GLCM approach. International journal of scientific and research publications, 3(5), 1-5.

15. Triantafyllou, D., Stavropoulos, G., \& Tzovaras, D. (2019, October). Iris Authentication Utilizing Cooccurrence Matrices and Textile Features. In 2019 International Conference on Speech Technology and Human-Computer Dialogue (SpeD) (pp. 1-6). IEEE.

16. Lesmana, I. P. D., Purnama, I. K. E., \& Purnomo, M. H. (2011, November). Abnormal condition detection of pancreatic beta-cells as the cause of diabetes mellitus based on iris image. In 2011 2nd International Conference on Instrumentation, Communications, Information Technology, and Biomedical Engineering (pp. 150-155). IEEE.

17. http://www.cbsr.ia.ac.cn/english/IrisDatabase.asp

18. https://www.researchgate.net/figure/Jensen-Charta-Right-iris_fig1_261211059 